Open Access

\title{
Associations of physical activity with depressiveness and coping in subjects with high-grade obesity aiming at bariatric surgery: a cross-sectional study
}

\author{
Ulf Elbelt ${ }^{1} 2^{*}$, Anne Ahnis ${ }^{2}$, Andrea Riedl ${ }^{2}$, Silke Burkert ${ }^{3}$, Tatjana Schuetz ${ }^{4}$, Juergen Ordemann ${ }^{5}$, \\ Christian J. Strasburger ${ }^{1}$ and Burghard F. Klapp ${ }^{2}$
}

\begin{abstract}
Background: Reduced physical activity is supposed to be associated with depressiveness and more passive coping patterns. For further evaluation of this assumed relation we studied energy expenditure due to physical activity - usually referred to as activity thermogenesis (AT) - together with depressiveness (clinical diagnosis, depression module of the Patient Health Questionnaire), and coping behaviours (Brief COPE Inventory) in 50 patients with high-grade obesity (42 \pm 12 years; 9 with $\|^{\circ}$ and 41 with $1 I^{\circ}$ obesity) aiming at bariatric surgery.
\end{abstract}

Methods: AT was assessed with a portable armband device (SenseWear ${ }^{\mathrm{TM}}$ armband). Depressiveness and coping were assessed using validated questionnaires.

Results: Weight-adjusted non-exercise AT and intensity of physical activity (metabolic equivalent) correlated inversely with body mass index (non-exercise AT: $r=-0.32, P<0.05$; mean metabolic equivalent: $r=-0.37, P<0.01$ ) but not with depressiveness. The coping strategies "support coping" and "active coping" showed significant inverse correlations to a) weight-adjusted non-exercise AT ("support coping": $r=-0.34, P<0.05$; "active coping": $r=-0.36, P<0.05$ ), b) weight-adjusted exercise-related AT ("support coping": $r=-0.36, P<0.05$; "active coping": $r=-0.38, P<0.01$ ) and c) intensity of physical activity (for mean metabolic equivalent: "support coping": $r=-0.38, P<0.01$; "active coping": $r=-0.40, P<0.01$; for duration of exercise-related AT: "support coping": $r=-0.36, P<0.05$; "active coping": $r=-0.38, P<0.01)$.

Conclusions: AT was not associated with depressiveness. Furthermore, supposed adaptive coping strategies of individuals aiming at bariatric surgery were negatively associated with AT.

Keyword: Bariatric surgery, Coping, Depression, Obesity, Physical activity

\section{Background}

Obesity is mainly caused by an imbalance of energy intake and energy expenditure [1] and leads to increased morbidity and mortality $[2,3]$. Total energy expenditure derives from resting energy expenditure which accounts for approximately $60 \%$ of it in sedentary or obese individuals

\footnotetext{
* Correspondence: ulf.elbelt@charite.de

'Department of Endocrinology, Diabetes and Nutrition, Charité - Universitätsmedizin Berlin, Charitéplatz 1, 10117 Berlin, Germany

${ }^{2}$ Charité Center for Internal Medicine and Dermatology, Division of General Internal and Psychosomatic Medicine, Charité - Universitätsmedizin Berlin, Charitéplatz 1, 10117 Berlin, Germany

Full list of author information is available at the end of the article
}

[4], energy expenditure due to physical activity and the thermogenic effect of food. Energy expenditure due to physical activity is usually referred to as activity thermogenesis (AT). Higher levels of physical activity leading to increased AT have been shown to improve weight loss and to be a good predictor of long-term weight loss maintenance in obese individuals [5]. AT can be further divided into exercise-related AT and non-exercise AT [4]. Of special interest is non-exercise AT (NEAT), i.e. AT during low- and moderate-intensity physical activities, as it is reported to be the most varying component of energy expenditure [6] and it is the quantitatively

\section{C)


more significant component of AT $[7,8]$. In a prospective study lower levels of non-exercise AT were shown to play an important role in the pathogenesis of obesity [8].

Higher body mass indices [9-12] and increased visceral fat [13-15] are frequently reported to be associated with depression or depressive symptoms, e.g. in a large statebased telephone survey in the US [9], adults with current depression (assessed in a telephone interview with the Patient Health Questionnaire 8 [PHQ-8]) or a history of diagnosed depression or anxiety by health care professionals were shown significantly more likely to be obese and physically inactive than those without this diagnosis [9]. Additionally, improvements in mood disturbance scores (assessed with the Profile of Mood States Short Form: negative mood, depression and tension) were associated with higher weight loss by severely obese women participating in a cognitive-behavioural exercise support treatment [16]. In another study achievement of a weight loss of $5 \mathrm{~kg}$ or more is reported to be more likely for obese women with symptoms of depression, if they are enrolled in a behavioural treatment program focusing on both weight loss and depression; this study showed an association of improvement in depression with an increase in self-reported physical activity [17]. In line with these findings, negative correlations of depression or depressive symptoms with physical activity $[9,11,18,19]$ or physical activity self-efficacy (i.e., an individual's confidence in his or her ability to manage physical activity behaviours) [10] have been reported. In addition, increases in physical activity proved protective against the onset of depressive symptoms $[20,21]$ and beneficial effects of physical activity on the course of depression have been described [17, 22, 23]. Therefore, physical activity is regarded as a preventive as well as a therapeutic treatment option in both conditions obesity and depression [24], resp. their co-morbidities.

The multimodal approaches for the conservative treatment of obesity (change in diet, modification of eating behaviour, increase of physical activity, cognitive behavioural therapy, relaxation techniques) focus on behavioural changes of obese individuals for achieving long-term weight loss [25]. Therefore, strategies of coping - commonly defined as cognitive and behavioural efforts used to manage external and internal demands [26] - play a central role in the treatment of obesity. In particular, obese individuals do not only have to cope with physical and mental consequences of obesity itself and with challenges of weight reduction attempts, but also with negative or discriminative social issues like stigmatization (obesity bias) [27]. However, for individuals with a body mass index (BMI) above $40.0 \mathrm{~kg} / \mathrm{m}^{2}$ or with a BMI between 35.0 and $39.9 \mathrm{~kg} / \mathrm{m}^{2}$ and co-morbidities such as type 2 diabetes and other metabolic disorders bariatric surgery is the most effective treatment in terms of long-lasting weight loss [25].
Coping includes strategies that help the individual to feel better, regardless of whether these strategies are positively adaptive (problem focused) or potentially dysfunctional [28] with respect to objective changes like weight loss. Successful behavioural factors for the achievement of long-term weight loss, like more initial weight loss, reaching the self-determined weight goal, gaining a physically active lifestyle, achieving a regular meal rhythm inclusive breakfast and healthier eating, control of over-eating, and self-monitoring of behaviours are listed in a comprehensive review by Elfhag and Rossner [29]. For successful maintenance of achieved weight loss psychological coping strategies must encompass high levels of self-efficacy, effective problem-focused coping strategies with stressful situations and the ability to generate coping responses for anticipated high risk scenarios (for relapse of overeating or physical inactivity) [30]. In contrast, weight re-gainers frequently report "escape-avoidance" coping such as eating, sleeping or wishing the problem would go away [30]. Rydén et al. [31] studied obesity-related coping in relation to treatment preference (surgery versus conventional treatment) in patients enrolled in the Swedish Obese Subjects intervention study. In their cohort of 2510 patients surgical candidates displayed lower scores for problemfocused (adaptive) coping ("social trust", "fighting spirit") and higher levels of emotion-focused (maladaptive) coping ("wishful thinking") than patients intending conventional therapy for weight loss [31].

Detailed information on direct relations of AT with depression, depressive symptoms, or coping styles of individuals with high-grade obesity aiming at bariatric surgery is not available, most likely due to difficulties in assessing AT and physical activity patterns precisely [32, 33]. However, modern technical devices like the SenseWear ${ }^{\mathrm{TM}}$ PRO 3 armband (BodyMedia, Inc., Pittsburgh, PA, USA) allow more reliable assessment of energy expenditure and physical activity patterns under ambulatory conditions [7, 34, 35].

In consequence of our previous finding that AT and intensity of physical activity gradually decline with rising weight categories from normal weight to subjects with III $^{\circ}$ obesity [7] we also assumed that within a cohort of obese individuals intending bariatric surgery AT would be more reduced and physical activity patterns would be further altered with increasing of obesity. We hypothesized that this assumed reduction of AT and physical activity would be correlated with the degree of depressiveness and a dominance of passive (maladaptive) coping patterns.

\section{Methods}

\section{Participants}

Fifty outpatients (10 men, 40 women, see Table 1) who presented for evaluation prior to bariatric surgery between spring 2008 and summer 2010 at Charité Universitätsmedizin Berlin by the Divisions of Clinical 
Endocrinology and General Internal and Psychosomatic Medicine were included in the study. The participants had a mean age of $42 \pm 12$ years (range 22-72 years). A medical history was taken and all participants underwent a complete physical examination. Weight was measured to the nearest $0.1 \mathrm{~kg}$ and height to the nearest $1 \mathrm{~cm}$. BMI was calculated as $\mathrm{kg} / \mathrm{m}^{2}$. The severity of obesity was classified according to the WHO criteria. Nine participants suffered from obesity $\mathrm{II}^{\circ}$ (BMI $35.0-39.9 \mathrm{~kg} / \mathrm{m}^{2}$ ) and 41 participants from obesity $\mathrm{III}^{\circ}\left(\mathrm{BMI} \geq 40.0 \mathrm{~kg} / \mathrm{m}^{2}\right)$. Endocrinological evaluation showed three patients suffering from subclinical (mild) hypothyroidism. However, another 15 participants were on replacement therapy with levothyroxine due to autoimmune hypothyroidism. One participant was treated with oral glucocorticoids due to a pseudolymphoma of the orbit leading to exophthalmos; clinical history and cortisol excretion did not indicate the presence of Cushing's syndrome in any of the patients. A psychosomatic evaluation (which in Germany is mandatory for cost coverage of bariatric surgery by the patients' health insurance companies) with the purpose of identifying factors opposing bariatric surgery or determining an appropriate point in time for bariatric surgery was performed and a set of questionnaires measuring psychological variables, like depressiveness and coping mechanisms, was administered on a personal digital assistant. Participants suffering from diseases hindering normal daily physical activities such as clinically relevant heart failure, limiting pulmonary diseases, severe osteoarthritis or amputations were not included in the study. All patients provided written informed consent for the scientific use of their data.

\section{Registration of energy expenditure and patterns of physical activity}

The SenseWear ${ }^{\mathrm{TM}}$ PRO 3 armband (BodyMedia, Inc., Pittsburgh, PA, USA) utilizes a multi-sensor array including a 2-axis accelerometer, heat flux sensor, galvanic skin response sensor, skin temperature sensor and a nearbody ambient temperature sensor for the estimation of total energy expenditure (TEE) and physical activity. Furthermore, with this device the degree of physical activity can be assessed and classified as either exercise-related AT (EAT) or non-exercise AT (NEAT) [7]. Therefore, we take the portable armband device for a suitable tool for the measurement of the different components of TEE. Data were analysed using a generalised proprietary algorithm developed by the manufacturer (InnerView ${ }^{\text {mi }}$ Professional, Version 6.1, BodyMedia, Inc., Pittsburgh, PA, USA).

Participants were asked to wear the armband at least $20.5 \mathrm{~h}$ per day. Physical activity was registered for three days (preferably 2 weekdays and 1 weekend day) with the portable armband device. While total energy expenditure (TEE) was directly received from the proprietary algorithms of the portable armband device, activity thermogenesis (AT) had to be calculated according to the equation: $\mathrm{AT}$ = total energy expenditure (TEE) - thermic effect of food - resting energy expenditure. The thermic effect of food was estimated with $10 \%$ of TEE and was calculated as TEE $\times 0.10$ [4]. Resting energy expenditure was calculated according to the equation of Müller et al. for obese individuals [36]: Resting energy expenditure $(\mathrm{MJ} / \mathrm{d})=0.05 \times$ weight $(\mathrm{kg})+1.103 \times$ gender $0.01586 \times$ age (years) +2.924 (for gender: female $=0$ and male $=1$ ). Then resting energy expenditure was converted to $\mathrm{kcal} / \mathrm{d}$.

Table 1 Demographic data, activity thermogenesis and patterns of physical activity

\begin{tabular}{|c|c|c|c|c|c|c|c|c|c|}
\hline & \multicolumn{2}{|l|}{ Obesity } & \multirow[t]{2}{*}{$P$} & \multicolumn{2}{|l|}{ Depression } & \multirow[t]{2}{*}{$P$} & \multicolumn{2}{|c|}{$\begin{array}{l}\text { Replacement therapy with } \\
\text { levothyroxine }\end{array}$} & \multirow[t]{2}{*}{$P$} \\
\hline & $\|^{\circ}(n=9)$ & \|\|$^{\circ}(n=41)$ & & No $(n=32)$ & Yes $(n=18)$ & & No $(n=35)$ & Yes $(n=15)$ & \\
\hline \multicolumn{10}{|l|}{ Demographic data: } \\
\hline Age, mean years $( \pm S D)$ & $46 \pm 16$ & $42 \pm 11$ & Ns & $41 \pm 13$ & $46 \pm 9$ & Ns & $42 \pm 11$ & $43 \pm 14$ & $\mathrm{~N}$ \\
\hline $\mathrm{BMl}$, mean kg/m² $( \pm \mathrm{SD})$ & $38 \pm 1$ & $48 \pm 6$ & * & $46 \pm 6$ & $46 \pm 7$ & Ns & $46 \pm 6$ & $46 \pm 8$ & $\Lambda$ \\
\hline \multicolumn{10}{|l|}{ Activity thermogenesis: } \\
\hline NEAT/kg, mean kcal/d ( \pm SD) & $8.3 \pm 3.1$ & $4.9 \pm 2.4$ & * & $5.3 \pm 2.2$ & $5.7 \pm 3.7$ & Ns & $5.6 \pm 3.0$ & $5.3 \pm 2.3$ & Ns \\
\hline EAT/kg, median kcal/d (quartiles) & $1.3(0.7 / 2.4)$ & $0.4(0.1 / 1.3)$ & Ns & $0.8(0.3 / 1.4)$ & $0.3(0.0 / 2.1)$ & Ns & $0.5(0.1 / 1.4)$ & $0.4(0.0 / 2.2)$ & $\mathrm{N}$ \\
\hline \multicolumn{10}{|l|}{ Physical activity: } \\
\hline Steps/d, mean ( \pm SD) & $7750 \pm 2153$ & $5867 \pm 2688$ & Ns & $6023 \pm 2459$ & $6532 \pm 3085$ & Ns & $6275 \pm 2712$ & $6044 \pm 2695$ & $\mathrm{Ns}$ \\
\hline MET, mean $( \pm \mathrm{SD})$ & $1.23 \pm 0.16$ & $1.03 \pm 0.16$ & * & $1.09 \pm 0.16$ & $1.04 \pm 0.20$ & Ns & $1.08 \pm 0.18$ & $1.05 \pm 0.17$ & Ns \\
\hline Duration of EAT, median min/d (quartiles) & $15(7 / 21)$ & $5(1 / 13)$ & * & $8(3 / 15)$ & $3(0 / 17)$ & Ns & $5(2 / 15)$ & $5(0 / 20)$ & $N$ \\
\hline
\end{tabular}

$B M I$ body mass index, EAT/kg exercise-related activity thermogenesis $/ \mathrm{kg}$ body weight, MET metabolic equivalent, $n$ number of participants, NEAT/kg non-exercise activity thermogenesis/kg body weight, Ns not significant, $S D$ standard deviation, ${ }^{*}: P<0.05$

Mean and standard deviation are given for parametric data, median and quartiles are given for non-parametric data. Two-tailed student t-tests for parametric data and Mann-Whitney-U-tests for non-parametric data were used 
In addition, the number of steps, metabolic equivalents (MET), and the duration of activity thermogenesis with distinct levels of physical activity graded in MET were directly obtained from the proprietary algorithms. MET define the energy expenditure related to body weight. One MET is equivalent to $1 \mathrm{kcal} / \mathrm{kg}$ body weight/hour. Energy expenditure of $>5$ MET was classified as exerciserelated $A T$ (EAT). Energy expenditure of $\leq 5 \mathrm{MET}$ was classified as non-exercise AT (NEAT) [7]. Lastly, NEAT was calculated as AT - EAT.

\section{Assessment of psychosocial dimensions}

As part of our psychosomatic assessment participants were individually interviewed with special attention for the presence and the degree of depressive symptoms by an experienced clinician. Depression was clinically diagnosed according to ICD-10 (International Classification of Diseases 10th Revision) by the interviewer and in case of diagnostic uncertainty after discussion by the psychosomatic team. In addition a set of questionnaires was presented with personal digital assistants (PDA):

Depressiveness In addition to the clinician's diagnosis, depressiveness was assessed with the 9-item depression module from the Patient Health Questionnaire (PHQ) which is a well validated and widely used screening instrument [37]. Sum scores may range from 0 to 27 with higher values indicating higher levels of depressiveness. The ICD-10-Symptom-Rating questionnaire (ISR) consists of 29 items based on relevant symptoms for the assessment of psychological disorders according to ICD-10 [38], as well as a "total score". The six syndrome scales include "depressive syndrome", "anxiety syndrome", "eating disorder syndrome", "obsessive syndrome", "somatoform syndrome", and a "supplementary scale", which covers a miscellaneous variety of syndromes. The ISR aims at a comprehensive evaluation of the severity of each disorder. Scores for the subscales and the total score range from 0 to 4 with higher values indicating a more severe syndrome [39].

Coping strategies Coping strategies were assessed with the German version of the Brief-COPE which consists of 28 items in four scales: "avoidant coping", "support coping", "positive reframing" and "active coping" [28]. This selfreporting questionnaire measures coping and explores strategies of handling difficult or awkward situations. Sum scores range from 6 to 24 for the scales "avoidant coping", "support coping", and "positive reframing". For the scale "active coping" it ranges from 4 to 16.

Assessment of further psychosocial dimensions Although our primary focus was the assessment of depressiveness and coping strategies we aimed at a more comprehensive psychosocial characterisation of our patients. Therefore, in addition, anxiety, physical complaints, and quality of life and mood were assessed. These instruments also served for testing the plausibility of clinical diagnosis of depression.

Anxiety Anxiety is frequently associated with depression and can often be considered as a symptom of depression [40]. The 7-item self-report Generalized anxiety disorder scale (GAD-7) was used [41], a well validated tool to screen for anxiety disorder and its severity. Sum scores range from 0 to 21 .

Physical Complaints Patients with depressive disorders often report an impaired physical state [42], in many cases bodily complaints are the most prominent symptoms. In this study, the subjective physical state was evaluated with a self-assessment questionnaire, the short version of Giessen Complaints Questionnaire (GBB-24) [43]. This well validated questionnaire consists of 24 items rated on a 5point response scale. The dimensions "exhaustion", "upper abdominal discomfort", "aching joints and muscles", "heart complaints" and a sum score for "pressure of complaints" were assessed. For a single dimension the scores range from 0 to 24, for the sum score from 0 to 96 .

Quality of life and mood Quality of life was assessed with the Short Form Health Survey (SF-8) [44] which is a 8-item version of the SF-36 questionnaire measuring the subjectively perceived health-related quality of life and recording the overall subjective state of health of adults for different diseases, in relation to their physical, psychological, and social aspects. Summary measures for physical condition and mental well-being are calculated by weighting each SF-8 item using a norm-based scoring method. Higher summary scores indicate better health. Mood was assessed with the Berlin Mood Questionnaire, which contains 30 items assigned to dimensions of e.g. "elated mood", "commitment", "anxious depressiveness", "tiredness", and "apathy" [45]. Mean scores range from 0 to 4 .

\section{Statistical analysis}

Results are expressed as mean and standard deviation (SD) for parametric data and as median and quartiles for non-parametric data. Two-tailed student t-tests for normally distributed data and Mann-Whitney-U-tests for non-normally distributed data were used. Pearson's coefficient of correlation was used to determine the degree of strength for the linear association between normally distributed variables and Spearman's coefficient of correlation was used for non-parametric variables. Stepwise regression analyses were used to identify the strongest predictors of energy expenditure components and physical activity measures. Statistical significance was set at $P<0.05$.

\section{Results}

Energy expenditure and patterns of physical activity Total energy expenditure (TEE) was $3356 \pm 562 \mathrm{kcal} / \mathrm{d}$. Resting energy expenditure (REE) was $2180 \pm 317 \mathrm{kcal} / \mathrm{d}$ 
according to the equation for obese individuals of Müller et al. [36]. Non-exercise AT (NEAT) was $707 \pm 317 \mathrm{kcal} / \mathrm{d}$ and exercise-related AT (EAT) was in median $73 \mathrm{kcal} / \mathrm{d}$ (quartiles: 16/206). The components of AT adjusted to body weight were: non-exercise AT/ $\mathrm{kg} 5.5 \pm 2.8 \mathrm{kcal} / \mathrm{kg} / \mathrm{d}$ and median exercise-related AT $/ \mathrm{kg} 0.5 \mathrm{kcal} / \mathrm{kg} / \mathrm{d}$ (quartiles: $0.1 / 1.4$ ). The number of daily steps was $6206 \pm 2681$ and metabolic equivalent (MET) $1.07 \pm 0.17$. Duration of exercise-related AT was at median $5 \mathrm{~min} / \mathrm{d}$ (quartiles: 2/15).

Participants with $\mathrm{III}^{\circ}$ obesity compared to participants with $\mathrm{II}^{\circ}$ obesity (see second and third part of Table 1) showed lower levels of body weight-adjusted NEAT, a lower average of MET, and a shorter daily duration of EAT. Contrary to our hypothesis, energy expenditure due to activity thermogenesis and patterns of physical activity did not differ between depressive and non-depressive patients. Patients with regular thyroid function $(n=32)$ or subclinical (mild) hypothyroidism $(n=3$, thyroid-stimulating hormone [TSH]: 4.18, 5.96, and $8.74 \mathrm{mU} / \mathrm{l}$ ) had a mean TSH of $2.30 \pm 1.69 \mathrm{mU} / \mathrm{l}$ (reference range: 0.3-4.0 mU/l) compared to participants on replacement therapy with levothyroxine $(n=15)$ with a mean TSH of $1.73 \pm 1.77 \mathrm{mU} / \mathrm{l}$ (two-tailed student $t$-test: $P=0.287$ ). As expected, mean free thyroxine (fT4) was higher with $1.14 \pm 0.19 \mathrm{ng} / \mathrm{dl}$ (reference range: $0.78-1.83 \mathrm{ng} / \mathrm{dl}$ ) for participants on replacement therapy compared with a mean fT4 of $1.01 \pm 0.20 \mathrm{ng} / \mathrm{dl}$ in patients with regular thyroid function or mild subclinical hypothyroidism (two-tailed student $t$-test: $P=0.026$ ). Components of energy expenditure and patterns of physical activity did not differ between participants with regular thyroid function and patients on levothyroxine replacement therapy (see Table 1).

\section{Results in the psychosocial dimensions}

By means of psychosomatic interviews 18 participants were clinically diagnosed to suffer from depression: in five cases a depressive episode (ICD-10: F32), one case of recurrent depressive disorder (ICD-10: F33), six cases of persistent affective disorder (dysthymia, ICD-10: F34.1), for one case "another affective disorder" (ICD-10: F38.8) was named, for one patient "mixed anxiety and depressive disorder" (ICD-10: F41.2) and for four patients "prolonged depressive reaction" due to an adjustment disorder (ICD-10: F43.2) were diagnosed. Eleven of these 18 participants were treated with (outpatient prescribed) antidepressants: one with opipramol, one with venlafaxine, three with citalopram, one with escitalopram, two with fluoxetine, two with sertraline, and one participant with paroxetine.

As depicted in Table 2, supporting the clinical diagnosis, patients with depression showed higher scores for depressiveness, psychosomatic co-morbidity (ISR-Scores for anxiety syndrome, obsessive syndrome, somatoform syndrome, supplementary scale, total score), higher anxiety score in GAD-7, more physical complaints (exhaustion, upper abdominal discomfort, aching joints and muscles, heart complaints, pressure of complaints), less mental wellbeing, less elated mood, and more negative mood like anxious depressiveness or tiredness. Coping strategies did not differ between patients with and without depression. Patients with obesity $\mathrm{II}^{\circ}$ or $\mathrm{III}^{\circ}$ did not differ in these psychosocial dimensions.

\section{Correlations of energy expenditure with age, body mass} index (see Fig. 1) and psychosocial dimensions (see Table 3)

Non-exercise AT and mean MET correlated negatively with body mass index (see Fig. 1), but did not correlate with depressiveness or the further psychosocial variables (psychosomatic co-morbidity, anxiety, physical complaints, quality of life and mood). The only psychological variables that correlated significantly with the physiological data here under investigation were the coping strategies "support coping" and "active coping", correlating negatively with body weight-adjusted non-exercise AT (NEAT) and the parameters for physical activity: body weight-adjusted exercise-related AT (EAT), mean MET, and duration of exercise-related AT (see Table 3).

\section{Stepwise regression analyses of energy expenditure, age,} body mass index and psychosocial dimensions

Due to intercorrelation of our variables we employed stepwise regression analysis and identified the coping strategy "active coping" as the most important predictor for body weight-adjusted NEAT (regression coefficient B -0.352 , standard-error 0.132, $P=0.010$ ), body weight-adjusted EAT (regression coefficient B -0.186 , standard-error 0.066, $P=0.007$ ), mean MET (regression coefficient B -0.020 , standard-error $0.008, P=0.015$ ) and duration of EAT (regression coefficient B -1.748 , standard-error $0.616, P=0.007)$. In addition, mean MET was also predicted by BMI (regression coefficient B -0.008 , standarderror $0.004, P=0.026)$. For the number of daily steps the strongest predictor was age (regression coefficient B -104.228, standard-error 28.077, $P=0.001$ ) followed by BMI (regression coefficient B -126.552 , standarderror 52.605, $P=0.020$ ).

\section{Discussion}

In accordance with the above reviewed literature (given in the section "Introduction") we assumed that a clinical diagnosis of depression or higher scores for depressiveness (assessed with validated questionnaires) and a dominance of passive (maladaptive) coping patterns would be associated with reduced AT and altered patterns of physical activity aggravating the degree of obesity. Furthermore, we assumed that these associations would be even more 
Table 2 Psychosocial dimensions

\begin{tabular}{|c|c|c|c|c|c|c|}
\hline & \multicolumn{2}{|l|}{ Obesity } & \multirow[t]{2}{*}{$P$} & \multicolumn{2}{|l|}{ Depression } & \multirow[t]{2}{*}{$P$} \\
\hline & $\|^{\circ}(n=9)$ & \|\|$^{\circ}(n=41)$ & & No $(n=32)$ & Yes $(n=18)$ & \\
\hline \multicolumn{7}{|c|}{ Measurement of depressiveness (PHQ, ISR): } \\
\hline PHQ - Depression module & $8.9 \pm 3.6$ & $10.1 \pm 5.9$ & Ns & $7.6 \pm 3.8$ & $14.0 \pm 6.0$ & $* *$ \\
\hline ISR - Depressive syndrome & $1.3(1.0 / 1.9)$ & $1.3(0.9 / 2.6)$ & Ns & $1.3(0.8 / 1.5)$ & $2.4(1.3 / 3.1)$ & $* *$ \\
\hline \multicolumn{7}{|c|}{ Measurement of coping strategies (Brief-COPE): } \\
\hline Avoidant coping & $11.7 \pm 3.3$ & $13.4 \pm 3.1(n=40)$ & Ns & $12.8 \pm 3.2$ & $13.5 \pm 3.2(n=17)$ & Ns \\
\hline Support coping & $11.9 \pm 3.3$ & $13.6 \pm 3.2(n=40)$ & Ns & $13.2 \pm 3.3$ & $13.4 \pm 3.4(n=17)$ & Ns \\
\hline Positive reframing & $10.0(8.5 / 17.0)$ & $11.0(10.0 / 12.8)(n=40)$ & Ns & $11.0(10.0 / 13.8)$ & $10.0(9.0 / 11.5)(n=17)$ & Ns \\
\hline Active coping & $11.1 \pm 3.2$ & $13.0 \pm 2.8(n=40)$ & Ns & $12.9 \pm 3.0$ & $12.1 \pm 2.6(n=17)$ & Ns \\
\hline
\end{tabular}

Measurement of additional psychosocial dimensions:

Measurement of psychosomatic co-morbidity (ISR):

\begin{tabular}{|c|c|c|c|c|c|c|}
\hline Anxiety syndrome & $0.8 \pm 0.9$ & $1.1 \pm 0.9$ & Ns & $0.8 \pm 0.7$ & $1.6 \pm 1.0$ & ** \\
\hline Eating disorder syndrome & $2.6 \pm 0.5$ & $2.3 \pm 0.8$ & Ns & $2.2 \pm 0.8$ & $2.6 \pm 0.6$ & Ns \\
\hline Obsessive syndrome & $0.0(0.0 / 0.8)$ & $0.0(0.0 / 0.8)$ & Ns & $0.0(0.0 / 0.7)$ & $0.7(0.0 / 1.3)$ & * \\
\hline Somatoform syndrome & $0.7(0.0 / 1.0)$ & $0.0(0.0 / 0.8)$ & Ns & $0.0(0.0 / 0.6)$ & $0.7(0.0 / 2.3)$ & * \\
\hline Supplementary scale & $0.7 \pm 0.4$ & $0.8 \pm 0.5$ & Ns & $0.6 \pm 0.4$ & $1.1 \pm 0.5$ & ** \\
\hline Total score & $1.0 \pm 0.4$ & $1.1 \pm 0.6$ & Ns & $0.8 \pm 0.3$ & $1.5 \pm 0.6$ & ** \\
\hline \multicolumn{7}{|l|}{ Measurement of anxiety (GAD-7): } \\
\hline Anxiety & $7.7 \pm 1.6$ & $8.4 \pm 5.4$ & Ns & $6.8 \pm 4.8$ & $10.9 \pm 4.2$ & ** \\
\hline \multicolumn{7}{|c|}{ Measurement of physical complaints (GBB-24): } \\
\hline Exhaustion & $11.1 \pm 4.3$ & $11.5 \pm 5.8$ & Ns & $9.7 \pm 4.2$ & $17.7 \pm 6.1$ & ** \\
\hline Upper abdominal discomfort & $5.2 \pm 3.5$ & $5.5 \pm 4.0$ & Ns & $3.8 \pm 2.8$ & $8.4 \pm 4.0$ & ** \\
\hline Aching joints and muscles & $13.0 \pm 6.2$ & $14.6 \pm 4.7$ & Ns & $12.8 \pm 4.0$ & $16.9 \pm 5.6$ & * \\
\hline Heart complaints & $6.9 \pm 3.9$ & $6.5 \pm 4.5$ & Ns & $5.0 \pm 3.3$ & $9.4 \pm 4.8$ & ** \\
\hline Pressure of complaints & $36.2 \pm 15.0$ & $38.2 \pm 16.1$ & Ns & $31.3 \pm 11.1$ & $49.4 \pm 16.5$ & ** \\
\hline \multicolumn{7}{|c|}{ Measurement of quality of life and mood (SF-8, Berlin Mood Questionnaire): } \\
\hline SF-8: Total physical score & $36.5 \pm 9.4$ & $32.3 \pm 9.4(n=40)$ & Ns & $33.9 \pm 9.8(n=31)$ & $31.6 \pm 8.9$ & Ns \\
\hline SF-8: Total mental score & $43.1 \pm 12.5$ & $45.7 \pm 13.2(n=40)$ & Ns & $50.2 \pm 10.4(n=31)$ & $36.6 \pm 12.8$ & ** \\
\hline Elated mood & $1.4 \pm 0.9$ & $1.4 \pm 0.9$ & Ns & $1.7 \pm 0.8$ & $1.0 \pm 0.8$ & * \\
\hline Commitment & $2.2 \pm 0.9$ & $2.3 \pm 0.8$ & Ns & $2.4 \pm 0.8$ & $2.1 \pm 0.9$ & Ns \\
\hline Anxious depressiveness & $1.2 \pm 0.9$ & $1.5 \pm 1.1$ & Ns & $1.1 \pm 0.9$ & $2.1 \pm 1.0$ & ** \\
\hline Tiredness & $2.0 \pm 0.5$ & $1.9 \pm 1.1$ & Ns & $1.6 \pm 0.8$ & $2.4 \pm 1.1$ & * \\
\hline Apathy & $0.2(0.0 / 1.3)$ & $0.4(0.0 / 1.0)$ & Ns & $0.3(0.0 / 0.8)$ & $0.4(0.2 / 1.4)$ & Ns \\
\hline
\end{tabular}

GAD-7 Generalized anxiety disorder scale, GBB-24 Short version of Giessen Complaints Questionnaire, ISR ICD-10-Symptom-Rating questionnaire, $n$ number of participants, Ns not significant, PHQ Patient Health Questionnaire, SD standard deviation, SF-8, Short Form Health Survey, *:P<0.05, ${ }^{* *}: P<0.01, * * *: P<0.001$ Mean and standard deviation are given for parametric data, median and quartiles are given for non-parametric data. Two-tailed student $t$-tests were used for parametric data and Mann-Whitney-U-tests for non-parametric data

pronounced in a cohort of patients suffering from such an extent of obesity that they aim at a bariatric surgery procedure.

\section{Obesity and energy expenditure due to physical activity} In line with observations previously reported $[7,46]$ we found the expected differences between participants with $\mathrm{II}^{\circ}$ or $\mathrm{III}^{\circ}$ obesity for activity thermogenesis and patterns of physical activity: Patients with $\mathrm{III}^{\circ}$ obesity spent significantly less energy in low- and moderateintensity physical activities (weight-adjusted non-exercise AT), showed a significantly lower mean metabolic equivalent (MET), and spent less time in intensive physical activities (weight-adjusted exercise-related AT). Additionally we found that the higher BMI was, the lower were body weight-adjusted NEAT and mean METs. 


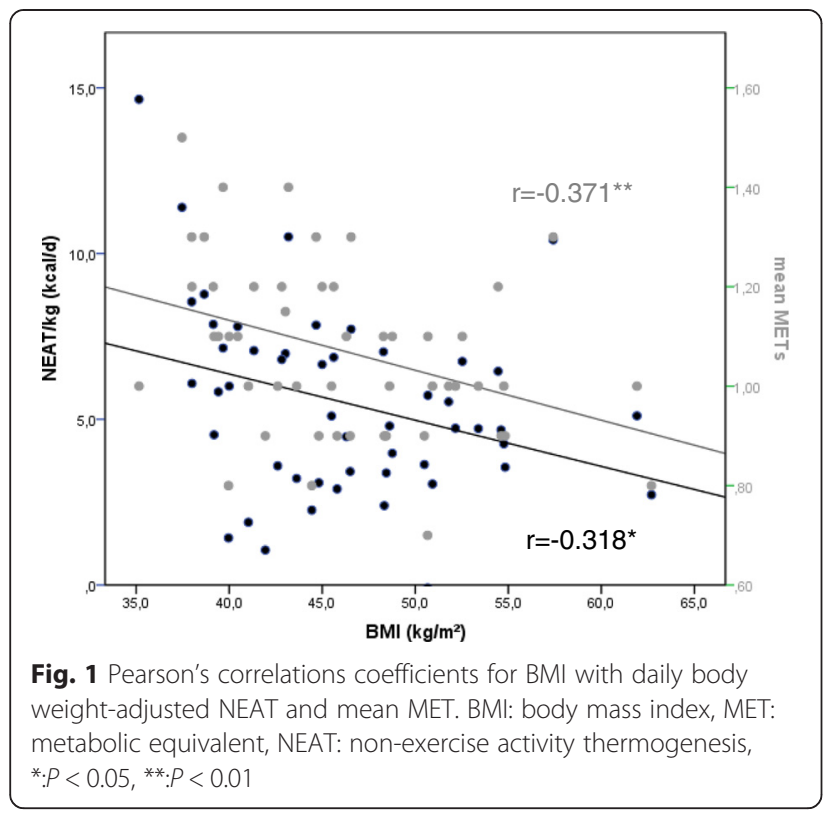

\section{Depressive symptoms and energy expenditure due to physical activity}

In contrast to reports of lower physical activity leading to or maintaining obesity in individuals suffering from depression [9, 16, 17], the hypothesised lower activity thermogenesis and altered physical activity patterns in patients with a diagnosis of (co-morbid) depression could not be proved.

An explanation for these conflicting results might be the difficulty of assessing energy expenditure: The abovementioned studies used physical activity recall questionnaires or interviews for quantifying physical activity in daily life
$[9,17]$. The inaccuracy of these activity logs is a major limitation in this approach. Four different questionnaires showed deviations up to $60 \%$ in comparison with the doubly labelled water method for the evaluation of physical activity [32]. In particular, obese participants more than normal weight individuals tend to overestimate their activity. Prince et al. addresses poor correlations between self-report and direct measures of physical activity [33]. Motion sensors including simple pedometers (measurement of steps) and technologically more advanced accelerometers (detection of body acceleration) provide objective approaches to assess physical activity. Tucker and Earl assessed emotional health (with the General Well-Being Schedule), energy intake (with food records) and physical activity using accelerometers (Actigraph ${ }^{\mathrm{Tx}}$ accelerometer) during a follow-up period of approximately 2 years in middle-aged women [47] and found that women with lower scores of emotional health seem to have a greater risk of weight gain. However, after adjustment for potential confounders this risk is not greater in depressed women than in their non-depressed counterparts.

We assume a high validity of our data concerning AT and physical activity patterns assessed with the portable armband device. However, one limitation might be that the time period of measurement might be too short as a distinct variation of physical activity has been described across the whole week with a decrease of moderate physical activity on Saturdays with rising weight category [34]. In order to overcome this limitation, we assessed energy expenditure preferably on 2 weekdays and 1 weekend day.

Another limiting aspect for interpretation of our data is the high percentage of depressed participants treated

Table 3 Association (correlation coefficients) of psychosocial dimensions with age, BMI, components of energy expenditure and patterns of physical activity

\begin{tabular}{|c|c|c|c|c|c|c|}
\hline & & \multicolumn{2}{|c|}{ Activity thermogenesis } & \multicolumn{3}{|c|}{ Physical activity } \\
\hline & & NEAT/kg & EAT $/ \mathrm{kg}$ & steps/d & mean MET & Duration of EAT \\
\hline \multicolumn{7}{|l|}{ Demographic data: } \\
\hline Age (years) & $r$ & -.114 & -.093 & $-.436^{* *}$ & -.155 & -.087 \\
\hline $\mathrm{BMI}\left(\mathrm{kg} / \mathrm{m}^{2}\right)$ & r & $-.318^{*}$ & -.214 & -.254 & $-.371^{* *}$ & -.225 \\
\hline \multicolumn{7}{|l|}{ Depressiveness (PHQ, ISR): } \\
\hline PHQ - Depression module & $r$ & .132 & -.020 & .023 & -.009 & -.055 \\
\hline ISR - Depressive syndrome & $r$ & .114 & -.138 & -.018 & -.026 & -.158 \\
\hline \multicolumn{7}{|l|}{ Coping strategies (Brief-COPE): } \\
\hline Avoidant coping & $r$ & .008 & -.088 & .026 & -.054 & -.121 \\
\hline Support coping & $r$ & $-.336^{*}$ & $-.356^{*}$ & .041 & $-.381^{* *}$ & $-.356^{*}$ \\
\hline Positive reframing & $r$ & -.191 & .049 & .081 & -.180 & .071 \\
\hline Active coping & $r$ & $-.363^{*}$ & $-.380^{* *}$ & -.078 & $-.395^{* *}$ & $-.383^{* *}$ \\
\hline
\end{tabular}

$B M I$ body mass index, EAT exercise-related activity thermogenesis, EAT/kg exercise-related activity thermogenesis/kg body weight, ISR ICD-10-Symptom-Rating questionnaire, MET metabolic equivalent, NEAT/kg non-exercise activity thermogenesis/kg body weight, $P H Q$ Patient Health Questionnaire, $r$ coefficient of correlation, *: $P<0.05,{ }^{* *}: P<0.01,{ }^{* * *}: P<0.001$

Pearson's coefficient of correlation was used for normally distributed variables and Spearman's coefficient of correlation was used for non-parametric variables 
with antidepressants (11 out of 18). Although participants with clinically diagnosed depression still show higher scores for depressiveness, we cannot exclude that the treatment with antidepressant drugs attenuates the hypothesised correlation between physical activity and depression.

Since we studied only individuals with high-grade obesity one might speculate that the decreases in AT and physical activity associated with body mass are so strong that they outweigh possible changes in energy expenditure associated with depression, which have been described before only in overweight individuals or those with low-grade obesity. This explanation is corroborated by findings of Hrabosky et al. in obese persons suffering from binge eating disorder [48]. Physical inactivity assessed with a self-report questionnaire (Paffenbarger Physical Activity Questionnaire) is correlated with BMI (mean BMI $38.2 \pm 5.7 \mathrm{~kg} / \mathrm{m}^{2}$ ) but not with the depressive affect as measured with the Beck Depression Inventory [48].

\section{Coping strategies and activity thermogenesis}

Rydén et al. described lower problem-focused and higher emotion-focused coping scores in candidates for bariatric surgery compared to individuals preferring a conventional treatment [31]. Similar results were reported by Hörchner et al. [49]. In our cohort higher scores for the supposed adaptive coping strategies "support coping" and "active coping" were accompanied by lower body weight-adjusted NEAT, lower exercise-related AT, lower mean MET, and shorter duration of exercise-related AT.

In the original version of the Brief-COPE the scale "active coping" was constructed by Carver as a problem-oriented approach to difficult situations [28]. However, for our cohort the scale "active coping" does not seem to assess problem-focused coping in the sense of leading to a necessary increase of physical activity. The "active coping" scale includes the following items: "I've been concentrating my efforts on doing something about the situation I'm in.";"I've been taking action to try to make the situation better"; "I've been thinking hard about what steps to take."; and "I've been trying to come up with a strategy about what to do.". The high scores may be explained by our patients' perception of being very active to achieve approval for bariatric surgery (e.g. participation in patient information events and support group meetings, preparation and submission of applications for cost coverage of bariatric surgery to the patients' health insurance company). This is in line with the finding recently described by Ahnis et al. [50] that patients prior to bariatric surgery showed higher scores for "active coping" (Brief-COPE) than obese patients participating in a 1-year multimodal outpatient weight reduction program.

\section{Conclusions}

We found that lower energy expenditure due to physical activity is associated with higher grades of obesity. However, our assumption of lower activity thermogenesis and of differences in physical activity patterns (like a lower number of daily steps, and a lower value of mean MET) in depressive obese individuals could not be confirmed. The coping strategies "active coping" and "support coping" interpreted in the sense of partly delegating the task of weight management to surgeons - were associated with lower body weight-adjusted activity thermogenesis.

Our findings highlight the enormous obstacles for high-grade obese patients to increase their physical activity which would be necessary for conservative achievement of long-lasting weight loss indicating bariatric surgery to be a more promising treatment option. These obstacles were less clearly associated with affective disturbances than was expected from reports in the literature (concerning degree of depressiveness, psychosomatic co-morbidity, anxiety, physical complaints, and quality of life and mood). However, it might be that the patients' decision to aim at bariatric surgery leads to under-reporting of affective and behavioural issues which might jeopardize their success in obtaining the surgical procedure.

\section{Abbreviations}

AT: Activity thermogenesis; BMI: Body mass index; EAT: Exercise-related activity thermogenesis; MET: Metabolic equivalent; NEAT: Non-exercise activity thermogenesis; REE: Resting energy expenditure; TEE: Total energy expenditure.

\section{Competing interests}

The authors declare that they have no competing interests.

\section{Authors' contributions}

UE participated in study design, data collection, data editing, statistical analysis and drafted this manuscript. AA, AR and SB participated in study design, data collection and discussion. TS was involved in study design and discussion. JO and CJS actively provided discussion and advice. BFK participated in study design, interpretation of data, discussion, manuscript preparation and also supervised all aspects of this study. All authors read, revised and approved the final manuscript.

\section{Acknowledgment}

The authors thank Vera Loening-Baucke for the critical review of the manuscript.

\section{Author details}

'Department of Endocrinology, Diabetes and Nutrition, Charité - Universitätsmedizin Berlin, Charitéplatz 1, 10117 Berlin, Germany. ${ }^{2}$ Charité Center for Internal Medicine and Dermatology, Division of General Internal and Psychosomatic Medicine, Charité - Universitätsmedizin Berlin, Charitéplatz 1, 10117 Berlin, Germany. ${ }^{3}$ Institute of Medical Psychology, Charité - Universitätsmedizin Berlin, Luisenstr. 57, 10117 Berlin, Germany. ${ }^{4}$ IFB Adiposity Diseases, Leipzig University Medical Center, Philipp-Rosenthal-Str. 27, 04103 Leipzig, Germany. ${ }^{5}$ Department of Surgery, Obesity Center, Charité - Universitätsmedizin Berlin, Charitéplatz 1, 10117 Berlin, Germany.

Received: 26 January 2015 Accepted: 10 June 2015

Published online: 25 June 2015

\section{References}

1. Thompson D, Karpe F, Lafontan M, Frayn K. Physical activity and exercise in the regulation of human adipose tissue physiology. Physiol Rev. 2012;92(1):157-91. 
2. Schmidt M, Johannesdottir SA, Lemeshow S, Lash TL, Ulrichsen SP, Bøtker $H E$, Sørensen HT: Obesity in young men, and individual and combined risks of type 2 diabetes, cardiovascular morbidity and death before 55 years of age: a Danish 33-year follow-up study. BMJ Open 2013,3e002698. doi: 10.1136/bmjopen-2013-002698.

3. Freedman DM, Ron E, Ballard-Barbash R, Doody MM, Linet MS. Body mass index and all-cause mortality in a nationwide US cohort. Int J Obes (Lond). 2006:30(5):822-9.

4. Levine JA. Non-exercise activity thermogenesis (NEAT). Best Pract Res Clin Endocrinol Metab. 2002;16(4):679-702.

5. Wing RR, Hill JO. Successful weight loss maintenance. Annu Rev Nutr. 2001;21:323-41.

6. Ravussin E, Lillioja S, Anderson TE, Christin L, Bogardus C. Determinants of 24-hour energy expenditure in man. Methods and results using a respiratory chamber. J Clin Invest. 1986;78(6):1568-78.

7. Elbelt U, Schuetz T, Hoffmann I, Pirlich M, Strasburger CJ, Lochs H. Differences of energy expenditure and physical activity patterns in subjects with various degrees of obesity. Clin Nutr. 2010;29(6):766-72.

8. Levine JA, Eberhardt $\mathrm{NL}$, Jensen MD. Role of nonexercise activity thermogenesis in resistance to fat gain in humans. Science. 1999;283(5399):212-4.

9. Strine TW, Mokdad AH, Dube SR, Balluz LS, Gonzalez O, Berry JT, et al. The association of depression and anxiety with obesity and unhealthy behaviors among community-dwelling US adults. Gen Hosp Psychiatry. 2008;30(2):127-37.

10. Konttinen H, Silventoinen K, Sarlio-Lähteenkorva S, Männistö S, Haukkala A. Emotional eating and physical activity self-efficacy as pathways in the association between depressive symptoms and adiposity indicators. Am J Clin Nutr. 2010;92(5):1031-9.

11. de Wit LM, Fokkema M, van Straten A, Lamers F, Cuijpers P, Penninx BW. Depressive and anxiety disorders and the association with obesity, physical, and social activities. Depress Anxiety. 2010;27(11):1057-65.

12. Dragan A, Akhtar-Danesh N. Relation between body mass index and depression: a structural equation modeling approach. BMC Med Res Methodol. 2007;7:17.

13. Everson-Rose SA, Lewis TT, Karavolos K, Dugan SA, Wesley D, Powell LH. Depressive symptoms and increased visceral fat in middle-aged women. Psychosom Med. 2009;71(4):410-6.

14. Rivenes AC, Harvey SB, Mykletun A. The relationship between abdominal fat, obesity, and common mental disorders: results from the HUNT study. J Psychosom Res. 2009;66(4):269-75.

15. Ahlberg AC, Ljung T, Rosmond R, McEwen B, Holm G, Akesson HO, Bjorntorp P. Depression and anxiety symptoms in relation to anthropometry and metabolism in men. Psychiatry Res. 2002;112(2):101-10.

16. Annesi JJ. Relations of mood with body mass index changes in severely obese women enrolled in a supported physical activity treatment. Obes Facts. 2008;1(2):88-92.

17. Simon GE, Rohde P, Ludman EJ, Jeffery RW, Linde JA, Operskalski BH, Arterburn D. Association between change in depression and change in weight among women enrolled in weight loss treatment. Gen Hosp Psychiatry. 2010;32(6):583-9.

18. Beydoun MA, Wang Y. Pathways linking socioeconomic status to obesity through depression and lifestyle factors among young US adults. J Affect Disord. 2010;123(1-3):52-63.

19. Teychenne M, Ball K, Salmon J. Associations between physical activity and depressive symptoms in women. Int J Behav Nutr Phys Act. 2008;5:27.

20. Ball K, Burton NW, Brown WJ. A prospective study of overweight, physical activity, and depressive symptoms in young women. Obesity (Silver Spring). 2009;17(1):66-71.

21. Strawbridge WJ, Deleger S, Roberts RE, Kaplan GA. Physical activity reduces the risk of subsequent depression for older adults. Am J Epidemiol. 2002;156(4):328-34

22. Fox KR. The influence of physical activity on mental well-being. Public Health Nutr. 1999;2(3A):411-8

23. Foreyt JP, Brunner RL, Goodrick GK, St Jeor ST, Miller GD. Psychological correlates of reported physical activity in normal-weight and obese adults: the Reno diet-heart study. Int J Obes Relat Metab Disord. 1995;19 Suppl 4:69-72

24. Blumenthal JA, Babyak MA, Moore KA, Craighead WE, Herman S, Khatri P, et al. Effects of exercise training on older patients with major depression. Arch Intern Med. 1999;159(19):2349-56.

25. Tsigos C, Hainer V, Basdevant A, Finer N, Fried M, Mathus-Vliegen E, Micic D, Maislos M, Roman G, Schutz Y, Toplak H, Zahorska-Markiewicz B. Management of obesity in adults: European clinical practice guidelines. Obes Facts. 2008;1(2):106-16.
26. Folkman S, Lazarus RS. The relationship between coping and emotion: implications for theory and research. Soc Sci Med. 1988;26(3):309-17.

27. Li W, Rukavina P. A review on coping mechanisms against obesity bias in physical activity/education settings. Obes Rev. 2009;10(1):87-95.

28. Carver CS. You want to measure coping but your protocol's too long: consider the brief COPE. Int J Behav Med. 1997:4(1):92-100.

29. Elfhag K, Rossner S. Who succeeds in maintaining weight loss? a conceptual review of factors associated with weight loss maintenance and weight regain. Obes Rev. 2005;6(1):67-85.

30. Byrne SM. Psychological aspects of weight maintenance and relapse in obesity. J Psychosom Res. 2002;53(5):1029-36.

31. Rydén A, Karlsson J, Persson LO, Sjöström L, Taft C, Sullivan M. Obesity-related coping and distress and relationship to treatment preference. $\mathrm{Br} J$ Clin Psychol. 2001;40(2):177-88.

32. Mahabir S, Baer DJ, Giffen C, Clevidence BA, Campbell WS, Taylor PR Hartman TJ. Comparison of energy expenditure estimates from 4 physical activity questionnaires with doubly labeled water estimates in postmenopausal women. Am J Clin Nutr. 2006;84(1):230-6.

33. Prince SA, Adamo KB, Hamel ME, Hardt J, Connor Gorber S, Tremblay M. A comparison of direct versus self-report measures for assessing physical activity in adults: a systematic review. Int J Behav Nutr Phys Act. 2008;5:56.

34. Scheers T, Philippaerts R, Lefevre J. Patterns of physical activity and sedentary behavior in normal-weight, overweight and obese adults, as measured with a portable armband device and an electronic diary. Clin Nutr. 2012;31(5):756-64.

35. Welk GJ, McClain JJ, Eisenmann JC, Wickel EE. Field validation of the MTI actigraph and body media armband monitor using the IDEEA monitor. Obesity (Silver Spring). 2007;15(4):918-28.

36. Müller MJ, Bosy-Westphal A, Klaus S, Kreymann G, Lührmann PM, NeuhäuserBerthold $\mathrm{M}$, et al. World health organization equations have shortcomings for predicting resting energy expenditure in persons from a modern, affluent population: generation of a new reference standard from a retrospective analysis of a German database of resting energy expenditure. Am J Clin Nutr. 2004:80(5):1379-90.

37. Spitzer RL, Kroenke K, Williams JB. Validation and utility of a self-report version of PRIME-MD: the PHQ primary care study. Primary care evaluation of mental disorders. Patient health questionnaire. JAMA. 1999;282(18):1737-44.

38. Tritt K, von Heymann F, Zaudig M, Zacharias I, Söllner W, Loew T. [Development of the "ICD-10-Symptom-Rating" (ISR) questionnaire]. Z Psychosom Med Psychother. 2008;54(4):409-18.

39. Fischer HF, Tritt K, Klapp BF, Fliege H. [Factor structure and psychometric properties of the ICD-10-Symptom-Rating (ISR) in samples of psychosomatic patients]. Psychother Psychosom Med Psychol. 2010;60(8):307-15.

40. Goldberg D, Fawcett J. The importance of anxiety in both major depression and bipolar disorder. Depress Anxiety. 2012;29(6):471-8

41. Spitzer RL, Kroenke K, Williams JB, Lowe B. A brief measure for assessing generalized anxiety disorder: the GAD-7. Arch Intern Med. 2006;166(10):1092-7.

42. Greden JF. Physical symptoms of depression: unmet needs. J Clin Psychiatry. 2003:64 Suppl 7:5-11.

43. Brähler E, Schumacher J, Brähler C. [First all-Germany standardization of the brief form of the Gissen complaints questionnaire GBB-24]. Psychother Psychosom Med Psychol. 2000;50(1):14-21.

44. Turner-Bowker DM, Bayliss MS, Ware Jr JE, Kosinski M. Usefulness of the SF-8 health survey for comparing the impact of migraine and other conditions. Qual Life Res. 2003;12(8):1003-12.

45. Hörhold M, Bolduan D, Klapp C, Volger H, Scholler G, Klapp B. TTesting a screening strategy for identifying psychosomatic patients in gynecologic practice]. Psychother Psychosom Med Psychol. 1997:47(5):156-62.

46. Rosemann T, Grol R, Herman K, Wensing M, Szecsenyi J. Association between obesity, quality of life, physical activity and health service utilization in primary care patients with osteoarthritis. Int J Behav Nutr Phys Act. 2008;5:4.

47. Tucker LA, Earl AB. Emotional health and weight gain: a prospective study of midlife women. Am J Health Promot. 2010;25(1):30-5.

48. Hrabosky Jl, White MA, Masheb RM, Grilo CM. Physical activity and its correlates in treatment-seeking obese patients with binge eating disorder. Int J Eat Disord. 2007:40(1):72-6.

49. Hörchner $\mathrm{R}$, Tuinebreijer WE, Kelder H, van Urk E. Coping behavior and Ioneliness among obese patients. Obes Surg. 2002;12(6):864-8.

50. Ahnis A, Figura A, Hofmann T, Stengel A, Elbelt U, Klapp BF. Surgically and conservatively treated obese patients differ in psychological factors, regardless of body mass index or obesity-related co-morbidities: a comparison between groups and an analysis of predictors. PLoS One. 2015;10(2):e0117460. 